\title{
Hydrocephalus Decreases Arterial Spin-Labeled Cerebral Perfusion
}

\author{
K.W. Yeom, R.M. Lober, A. Alexander, S.H. Cheshier, and M.S.B. Edwards
}

\section{ABSTRACT}

BACKGROUND AND PURPOSE: Reduced cerebral perfusion has been observed with elevated intracranial pressure. We hypothesized that arterial spin-labeled CBF can be used as a marker for symptomatic hydrocephalus.

MATERIALS AND METHODS: We compared baseline arterial spin-labeled CBF in 19 children (median age, 6.5 years; range, 1-17 years) with new posterior fossa brain tumors and clinical signs of intracranial hypertension with arterial spin-labeled CBF in 16 age-matched controls and 4 patients with posterior fossa tumors without ventriculomegaly or signs of intracranial hypertension. Measurements were recorded in the cerebrum at the vertex, deep gray nuclei, and periventricular white matter and were assessed for a relationship to ventricular size. In 16 symptomatic patients, we compared cerebral perfusion before and after alleviation of hydrocephalus.

RESULTS: Patients with uncompensated hydrocephalus had lower arterial spin-labeled CBF than healthy controls for all brain regions interrogated $(P<.001)$. No perfusion difference was seen between asymptomatic patients with posterior fossa tumors and healthy controls $(P=1.000)$. The median arterial spin-labeled CBF increased after alleviation of obstructive hydrocephalus $(P<.002)$. The distance between the frontal horns inversely correlated with arterial spin-labeled CBF of the cerebrum $(P=.036)$ but not the putamen $(P=.156)$, thalamus $(P=.111)$, or periventricular white matter $(P=.121)$.

CONCLUSIONS: Arterial spin-labeled-CBF was reduced in children with uncompensated hydrocephalus and restored after its alleviation. Arterial spin-labeled-CBF perfusion MR imaging may serve a future role in the neurosurgical evaluation of hydrocephalus, as a potential noninvasive method to follow changes of intracranial pressure with time.

ABBREVIATIONS: $A S L=$ arterial spin-labeled; $I C P=$ intracranial pressure; $P F=$ posterior fossa

$\mathbf{H}$ ydrocephalus is a common neurosurgical condition in children and adults, accounting for approximately 69,000 annual hospital admissions and 39,000 shunt procedures in the United States. ${ }^{1,2}$ While concepts of hydrocephalus remain complex, including pathophysiology, diagnostic and therapeutic approaches, ${ }^{3-5}$ and outcome, ${ }^{6,7}$ it is generally accepted that hydrocephalus reflects pathologic dynamics among brain, spinal cord, blood, and CSF within a confined environment. ${ }^{8-10}$ In clinical practice, imaging is

Received October 22, 2013; accepted after revision November 13.

From the Departments of Radiology (K.W.Y.) and Neurosurgery (R.M.L., A.A., S.H.C., M.S.B.E.) and Division of Pediatric Neurosurgery (S.H.C., M.S.B.E.), Lucile Packard Children's Hospital at Stanford University, Palo Alto, California.

Paper was previously presented in part in abstract form at: Meeting of American Association of Neurological Surgeons, April 14-18, 2012, Miami, Florida; and Annual Meeting of the Society for Pediatric Radiology, April 16-17, 2012; San Francisco, California.

Please address correspondence to Kristen W. Yeom, MD, Department of Radiology, Lucile Packard Children's Hospital, Stanford University, Pediatric MRI \& CT, Room 0511, 725 Welch Rd, Palo Alto, CA 94304; e-mail: kyeom@stanford.edu

Indicates article with supplemental on-line figures.

http://dx.doi.org/10.3174/ajnr.A3891 often used to work-up hydrocephalus in search of obstructing lesions or the presence of ventriculomegaly. However, ventricular size, a frequently used imaging feature, does not always correlate with underlying CSF pressures or resorptive capacity for $\mathrm{CSF}^{11-16}$ and, therefore, may not reliably identify compensated hydrocephalus without signs of raised intracranial pressure (ICP) and progressive hydrocephalus with raised ICP.

Prior studies have shown reduced CBF with elevated ICP in various animal models of hydrocephalus. ${ }^{17-20}$ Reduced CBF has also been reported in small case series of children with either congenital hydrocephalus or acute hydrocephalus from brain tumors by using ${ }^{15} \mathrm{O}_{-\mathrm{PET}^{21}}$ or SPECT. ${ }^{22}$ Recently, 2D phase-contrast MRA has shown that carotid and basilar arterial flow rates are reduced in infants with hydrocephalus. ${ }^{23}$ However, $2 \mathrm{D}$ phase-contrast MRA does not directly measure $\mathrm{CBF}$ at the tissue level and may be hampered by long scanning times and flow-sensitive technical challenges. ${ }^{24,25}$

In contrast, arterial spin-labeled (ASL) MR imaging perfusion directly measures tissue perfusion without requiring long scanning times, contrast, or radioisotope injection. ${ }^{26}$ It is also 
uniquely advantageous in children with high labeling efficiency and SNR and can be repeated in the event of patient motion or after CSF diversion without the risk of radiation exposure. ${ }^{27}$ While ASL perfusion has increasingly become clinically available, no studies have investigated its role for evaluating hydrocephalus. We hypothesized that ASL-CBF is reduced in uncompensated hydrocephalus and improved after its alleviation and, therefore, can be used as a marker for symptomatic hydrocephalus.

\section{MATERIALS AND METHODS}

\section{Patients with Symptomatic Hydrocephalus}

All patients presenting with a posterior fossa (PF) brain tumor at our children's hospital from 2010 to August 2013 were retrospectively reviewed after approval by the institutional board (protocol 28674). The study cohort was identified by using the following inclusion criteria: The patients underwent a baseline ASL perfusion MR imaging at 3T, presented acutely with clinical signs of elevated ICP that required neurosurgical intervention (tumor resection or CSF diversion) within 7 days of a baseline ASL scan, had ventriculomegaly, and received no prior medical/radiation therapy or neurosurgical intervention, including CSF diversion.

One of the important pathogenic factors in hydrocephalus is increase in resistance to outflow of CSF, which has been correlated with elevated CSF pressure. ${ }^{11,14}$ Children acutely presenting with uncompensated hydrocephalus from a new PF tumor that required symptom alleviation were selected as our study cohort, given that this group represented a relatively uniform cohort with similar obstructive mechanisms and could be distinguished from those with chronic compensated or uncompensated hydrocephalus.

Patients with underlying cardiac disease, epilepsy, migraines, hemorrhage, vascular lesions (aneurysm, AVM, fistula, or stenoocclusive disease), prior strokes, concomitant supratentorial tumors or leptomeningeal seeding, and prior history of radiation or chemotherapy were excluded, given their potential impact on cerebral perfusion.

\section{Asymptomatic Patients}

Patients with an incidentally discovered PF tumor but without signs of elevated ICP based on neurosurgical assessment and without ventriculomegaly or interstitial periventricular edema on MR imaging were also included for comparison if they had undergone a treatment-naïve ASL perfusion MR imaging at 3T.

\section{Controls}

Healthy controls who underwent ASL perfusion at 3T as part of routine brain MR imaging were randomly selected from our data base and matched for age and sedation status of the study subjects. The control group consisted of children with no underlying neurologic diseases and normal brain MR imaging findings. Examples of clinical reasons for undergoing MR imaging were isolated headaches, cholesteatoma of the middle ear, isolated facial lesions (eg, hemangioma, dermoid), scalp nevus, inflammatory nasal obstruction, short stature, and family history of aneurysms. Children with neurocutaneous syndromes; ataxia; gaze abnormality; developmental delay; endocrinopathies; psychiatric diseases; migraines; and any chronic diseases, including epilepsy, cancer, and previously treated neurologic diseases; and prior or ongoing medical therapy were excluded.

\section{Clinical Assessment}

Neurosurgical documentation regarding patient symptoms at baseline and after neurosurgical intervention was reviewed, including the patient's clinical status at follow-up ASL perfusion and, if present, the functional status of the shunt or ventriculostomy.

\section{Imaging Methods}

All subjects underwent brain MR imaging at 3T (Discovery 750; GE Healthcare, Milwaukee, Wisconsin), by using an 8-channel head coil. The technique used to perform perfusion ASL MR imaging has been described in detail elsewhere. ${ }^{28}$ Briefly, our vendor-supplied ASL was performed by using a pseudocontinuous labeling period of $1500 \mathrm{~ms}$, followed by a 1500 -ms postlabel delay. Whole-brain images were acquired with a 3D background-suppressed fast spin-echo stack-of-spirals method, with a TR of approximately 5 seconds. Multiarm spiral imaging was used, with 8 arms and 512 points acquired on each arm (bandwidth, 62.5 $\mathrm{kHz}$ ), yielding in-plane and through-plane spatial resolutions of 3 and $4 \mathrm{~mm}$, respectively. A high level of background suppression was achieved by 4 separate inversion pulses spaced around the pseudocontinuous labeling pulse. The sequence required 5 minutes to acquire, which included proton-attenuation images required for CBF quantitation. For graphic prescription of the ASL, the sagittal image following the 3-plane localizer was used for alignment. Postprocessing was performed by using an automated reconstruction script that returned $\mathrm{CBF}$ images directly to the scanner console within 1 minute, by using the microsphere methodology described by Buxton et al. ${ }^{29}$ Other ASL MR imaging parameters were TR/TE, 4632/10.5 ms; FOV, $24 \mathrm{~cm}$; matrix, $512 \times$ 8 ; and NEX, 3.

\section{Imaging Analysis}

ASL Perfusion. Quantitative mean cerebral CBF was measured and averaged at 3 consecutive axial images just above the centrum semiovale near the vertex by using region-of-interest analysis (size, $6500 \mathrm{~mm}^{2}$ ). The axial planes near the vertex were selected, given a higher proportion of gray matter in this region, and because higher SNR is seen in the gray matter compared with white matter. ${ }^{30,31}$ Also, a large region-of-interest size was chosen in this region for a more global CBF assessment that is easy to perform in a clinical setting by a radiologist or a neurosurgeon. In addition, more specific anatomic regions were interrogated by using region of interest (size, $85 \mathrm{~mm}^{2}$ ) at the bilateral basal ganglia (at the level of putamina), thalami, and periventricular white matter. Examples are shown in Fig 1 . The ROIs were independently performed by 2 neurosurgeons (A.A., R.M.L.) blinded to clinical information, including the status of hydrocephalus and follow-up clinical management. A blinded pediatric neuroradiologist (K.W.Y.) with a Certificate of Added Qualification in neuroradiology (7 years of experience) confirmed the proper region-of-interest placement.

For comparison between CBF at baseline and after neurosurgical intervention, the last follow-up ASL perfusion scan at $3 \mathrm{~T}$ before initiation of any medical/radiation therapy was used so 

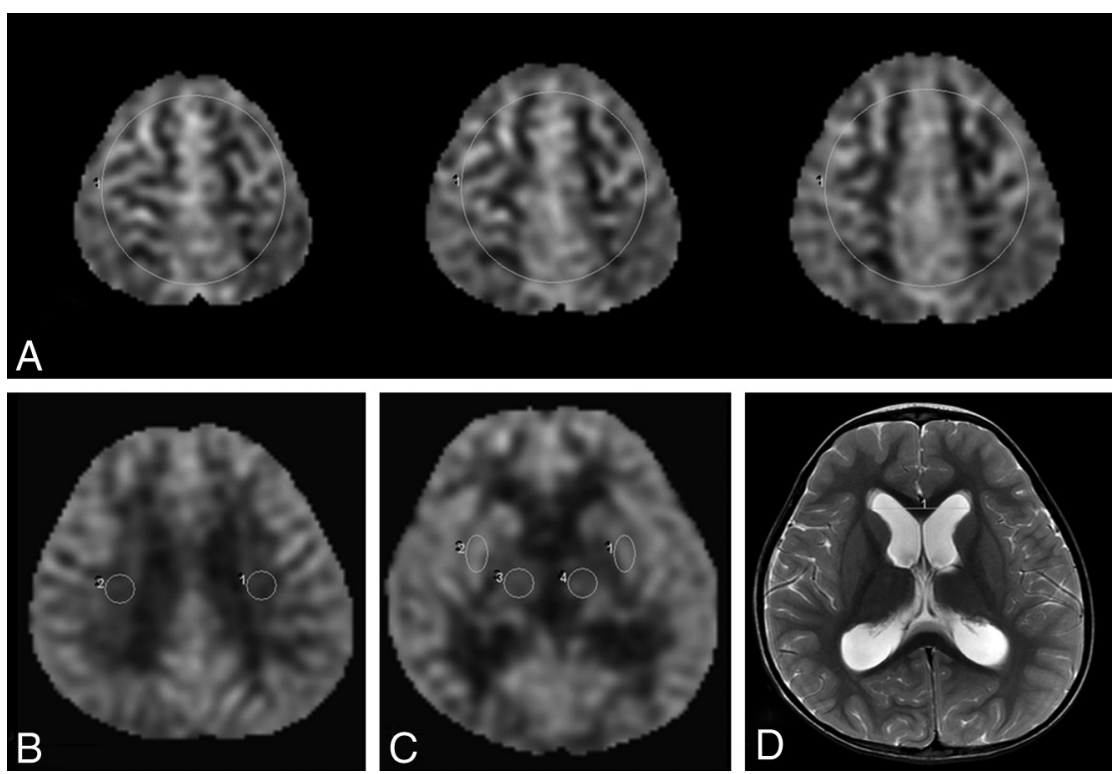

FIG 1. Brain region-of-interest placement and ventricular measurement. $A$, ROIs were placed in the 3 axial levels of the cerebrum near the vertex and just above the centrum semiovale. $B$ and $C$. Additional ROls were placed in bilateral periventricular white matter, and the deep gray matter, including the bilateral thalami and putamina. $D$, Ventricular measurement of the bilateral frontal horns was performed at the level of the caudate heads.

Table 1: Patient characteristics $(n=23)$

\begin{tabular}{lc}
\hline \multicolumn{1}{c}{ Characteristic } \\
\hline Age at initial diagnosis (yr) \\
Median \\
$\quad$ Range & 6.2 \\
Sex & $0.9-18$ \\
$\quad$ Male & $17(74 \%)$ \\
Female & $6(26 \%)$ \\
Diagnosis (in order of frequency) & \\
$\quad$ Medulloblastoma & $8(35 \%)$ \\
Pilocytic astrocytoma & $5(22 \%)$ \\
Ependymoma & $5(22 \%)$ \\
$\quad$ Choroid plexus papilloma & $2(9 \%)$ \\
Tectal astrocytoma & $2(9 \%)$ \\
$\quad$ Diffuse intrinsic pontine glioma & $1(5 \%)$ \\
Hydrocephalus at presentation & $19(83 \%)$ \\
\hline
\end{tabular}

that no therapy other than tumor decompression or CSF diversion occurred in the interval between the baseline and the follow-up MR imaging.

\section{Ventricular Size and Edema}

The bifrontal diameter at the level of the caudate heads and underlying perfusion were measured in each patient at baseline and serial follow-up MR imaging by a neuroradiologist (K.W.Y.) blinded to patient clinical status (symptomatic versus asymptomatic with regard to hydrocephalus) at the time of imaging. The presence or lack of periventricular interstitial edema was noted based on T2-weighted images.

\section{Statistical Analysis}

All statistical analyses were performed with the Statistical Package for the Social Sciences, Version 20.0 (IBM, Armonk, New York) with an a priori significance level of $\alpha=.05$. Associations of perfusion at various sites (eg, thalamus, putamen, and white matter) with age and diameter between the frontal ventricular horns were tested by using the 2-tailed Pearson correlation. Comparisons of perfusion between sexes and between patients with and without interstitial edema were made by using the Mann-Whitney $U$ test for independent samples. Comparisons of perfusion between healthy controls and asymptomatic patients with tumor and those symptomatic from obstructive hydrocephalus, based on tumor type, were made by using the Kruskal-Wallis test for independent samples with pair-wise comparisons. Differences between perfusion before and after relief of obstructive hydrocephalus were evaluated with the Wilcoxon signed rank test for related samples.

\section{RESULTS}

\section{Clinical Findings}

Twenty-three patients, median age, 6.2 years (range, 0.9-18 years), were included in the study. Patient demographics and clinical data are shown in Table 1. The control group matched by age and sedation status consisted of 16 children ( 7 boys and 9 girls; median age, 8.2 years; range, $1-18$ years).

\section{Patients with Symptomatic Hydrocephalus}

Nineteen patients with PF tumor presented with symptomatic obstructive hydrocephalus, 16 of whom underwent at least partial resection. In 6 of these patients, CSF diversion in the form of an extraventricular drain or ventriculoperitoneal shunt was performed at the time of the surgery or subsequent to tumor resection. Three patients with unresectable brainstem tumors also underwent CSF diversion: ventriculoperitoneal shunt placement in 1 case of diffuse intrinsic pontine glioma and endoscopic third ventriculostomy in 2 cases of tectal astrocytoma.

Seventeen patients demonstrated symptomatic improvement after removal of the obstructing mass and/or CSF diversion. Two patients continued to demonstrate intractable hydrocephalus after tumor resection either from shunt complications or extraventricular drain malfunction that required further surgery.

\section{Asymptomatic Patients}

Four patients with PF tumor were asymptomatic for hydrocephalus at presentation. These patients underwent MR imaging for neurofibromatosis type 1 , whole-body fever work-up, possible Bell palsy, and chronic headaches. Three of these patients electively underwent surgical resection of the tumor and 1 underwent focal CyberKnife.

\section{Sedation Status}

Sedation status was matched for all patients and their respective age-matched controls. For all patients with symptomatic hydrocephalus who underwent follow-up ASL perfusion, the sedation 
Table 2: Cerebral blood flow in healthy controls $(n=16)$ and patients with PF Tumor $(n=23)$

\begin{tabular}{llccc}
\hline & \multicolumn{3}{c}{ Median Cerebral Blood Flow (mL/100 g/min) (Range) } \\
\cline { 2 - 5 } & \multicolumn{1}{c}{ Cerebrum } & Basal Ganglia & Thalamus & White Matter \\
\hline Healthy control & $62.3(50.4-68.9)$ & $57.3(44.6-70.8)$ & $56.4(45.4-67.8)$ & $37.3(26-43.4)$ \\
PF tumor without hydrocephalus & $56.7(46.4-63.1)$ & $57.4(49.7-60.0)$ & $59.7(53.2-65.4)$ & $32.3(20.8-35.8)$ \\
PF tumor with hydrocephalus & $34.3(8.6-59.0)$ & $39.8(12.5-59.6)$ & $32.4(8.1-50.5)$ & $17.7(6.0-28.1)$ \\
\hline
\end{tabular}

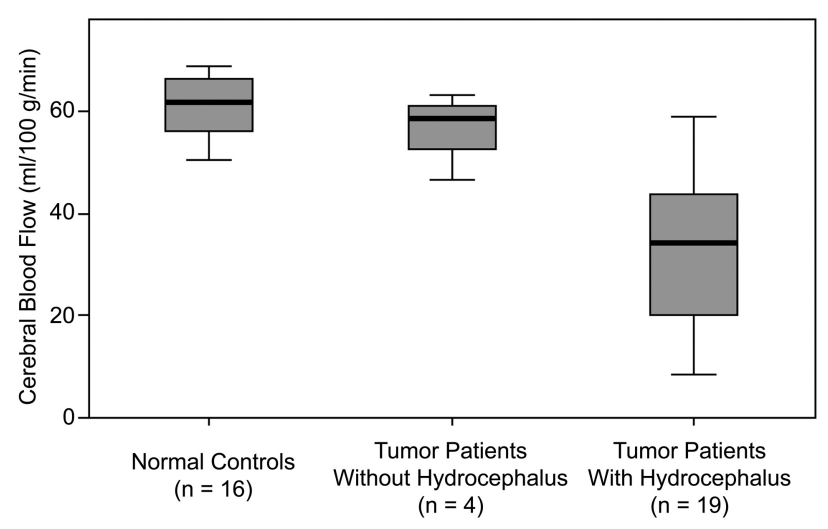

FIG 2. Comparison of ASL-CBF among controls, asymptomatic patients, and patients with hydrocephalus.

status was also identical for both baseline and follow-up MR imaging.

\section{Cerebral Blood Flow}

Controls. For the 16 age-matched and sedation-matched healthy controls, there was no correlation between age and CBF of the cerebrum at the vertex $(P=.884)$. Similarly, there were no correlations between age and perfusion at more specific sites - that is, the putamen $(P=.920)$, thalamus $(P=.244)$, or periventricular white matter $(P=.297)$.

Baseline CBF. CBF for all interrogated cerebral regions is summarized in Table 2. Patients with symptomatic hydrocephalus had lower $\mathrm{CBF}$ than healthy controls for all brain regions interrogated $(P<.001)$ (Fig 2). No perfusion difference was seen between asymptomatic patients with $\mathrm{PF}$ tumor and healthy controls $(P=1.000)$.

\section{Baseline and Follow-Up Comparison}

Of 17 patients who showed symptomatic relief after neurosurgical intervention, 16 had both baseline and follow-up ASL perfusion. The median time between intervention and follow-up MR imaging used in the comparison analysis was 27 days (range, 1 day to 7 months). The median CBF increased after alleviation of obstructive hydrocephalus $(P<.002)$ (Table 3 and Fig 3$)$. Examples are shown in Figs 4 and 5.

Two patients continued to demonstrate elevated ICP after tumor resection due to ventriculoperitoneal shunt complications (peritoneal contamination from gastrostomy) and extraventricular drain malfunction that required a new shunt placement or revision. Therefore, these patients were only included in the preintervention $\mathrm{CBF}$ analysis. For these 2 patients, $\mathrm{CBF}$ values were low during elevated ICP. For example, the patient with elevated ICP due to shunt complications from peritoneal contamination showed further decrease in perfusion with CBF values of 10, 15,
12 , and $6 \mathrm{~mL} / 100 \mathrm{~g} / \mathrm{min}$ at the cerebral vertex, putamen, thalamus, and periventricular white matter, respectively. MR imaging performed several hours after shunt revision showed mild improvement with CBF of $21,33,33$, and $13 \mathrm{~mL} / 100 \mathrm{~g} / \mathrm{min}$ in the respective brain regions. The other patient also showed low CBF during extraventricular drain malfunction with improvement after a new shunt placement (Fig 6).

\section{Ventricular Size and Other Parameters}

The bifrontal ventricle size ranged from 35 to $65 \mathrm{~mm}$ in our patients with hydrocephalus at presentation. $\mathrm{CBF}$ at each site did not correlate with $\operatorname{sex}(P>.082)$ or tumor type $(P>.203)$. Among the patients with tumor, the distance between the frontal horns inversely correlated with $\mathrm{CBF}$ of the cerebrum $(P=.036)$, but not the putamen $(P=.156)$, thalamus $(P=.111)$, or periventricular white matter $(P=.121)$.

Among patients with clinical improvement following relief of symptomatic hydrocephalus, ventricular size showed a small but statistically significant decrease, with a median change of $0.8 \mathrm{~mm}$ (range, $0.4-13.3 \mathrm{~mm})(P=.002)$.

\section{DISCUSSION}

Reduced ASL-CBF was seen in children presenting with acute hydrocephalus from PF tumors compared with controls, with restoration of CBF close to the normal range after alleviation of hydrocephalus. Prior studies have shown that when ICP increases, disturbance in autoregulatory vasomotor capacity can reduce cerebral perfusion pressure, where a pronounced decrease in CBF can result at cerebral perfusion pressure below 40-50 mm Hg. ${ }^{17,18}$ Because CBF was not significantly reduced in patients with PF tumors but without signs of raised ICP, CBF changes in our study were likely driven primarily by ICP effects rather than the presence of a mass lesion.

Infants and young children with hydrocephalus often present with nonspecific symptoms ${ }^{32}$; therefore, the distinction between ventriculomegaly and hydrocephalus with increased ICP is critically important. Given that many different etiologies for ventriculomegaly (underdevelopment, atrophy, metabolic diseases, and others) exist and ventricular size does not reliably identify symptomatic hydrocephalus, ${ }^{11-14,16}$ a method that reflects alterations in cerebral hydrohemodynamics is desired. To our knowledge, this is the first study to report the use of ASL perfusion as a marker for symptomatic hydrocephalus. In contrast to nuclear medicine, CT and MR imaging contrast perfusion, or 2D phase-contrast MRA methods, ASL perfusion does not require contrast or radiation, provides quantitative CBF measurements directly at the tissue level, and is technically easy to acquire at a relatively short scan time.

The CBF of our hydrocephalus cohort $(34.3 \pm 14.9 \mathrm{~mL} / 100$ $\mathrm{g} / \mathrm{min}$ ) is consistent with a prior report by Hayashi et $\mathrm{al},{ }^{33}$ which 
Table 3: Cerebral blood flow before and after alleviation of obstructive hydrocephalus $(n=16)^{a}$

\begin{tabular}{llccc}
\hline & \multicolumn{3}{c}{ Median Cerebral Blood Flow (mL/100 mg/min) (Range) } \\
\cline { 2 - 5 } & \multicolumn{1}{c}{ Cerebrum } & Basal Ganglia & Thalamus & White Matter \\
\hline Before alleviation of hydrocephalus & $35.8(8.6-59)$ & $38.0(12.5-59.6)$ & $29.9(8.1-50.3)$ & $17.5(6.0-28.1)$ \\
After alleviation of hydrocephalus & $52.6(29.8-69.7)$ & $53.9(40.2-68.8)$ & $55.3(29.1-63.3)$ & $31(23.4-45.4)$ \\
\hline
\end{tabular}

${ }^{a}$ For each site, CBF was increased following alleviation of hydrocephalus (Wilcoxon signed rank test for related samples, $P<.002$ ).

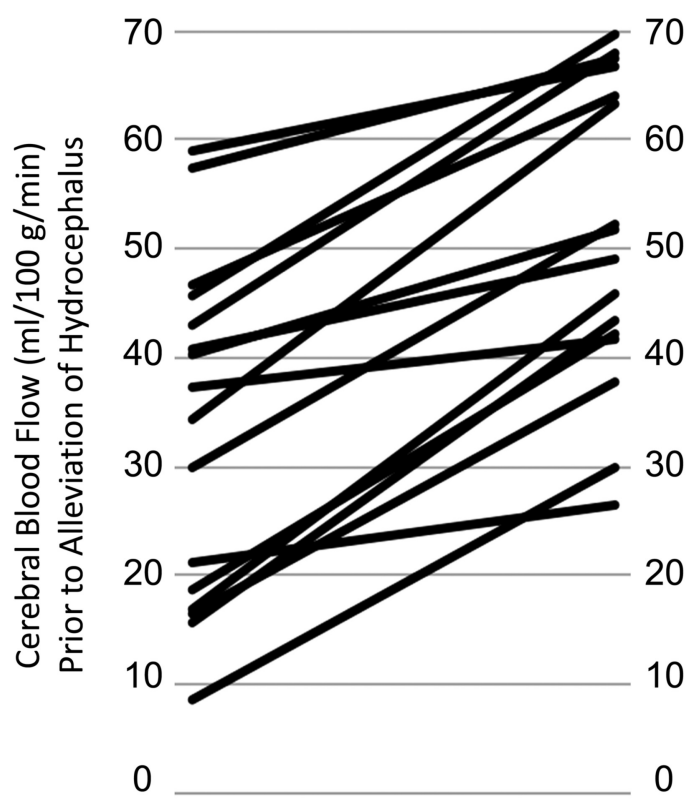

FIG 3. ASL-CBF values before and after alleviation of obstructive hydrocephalus.
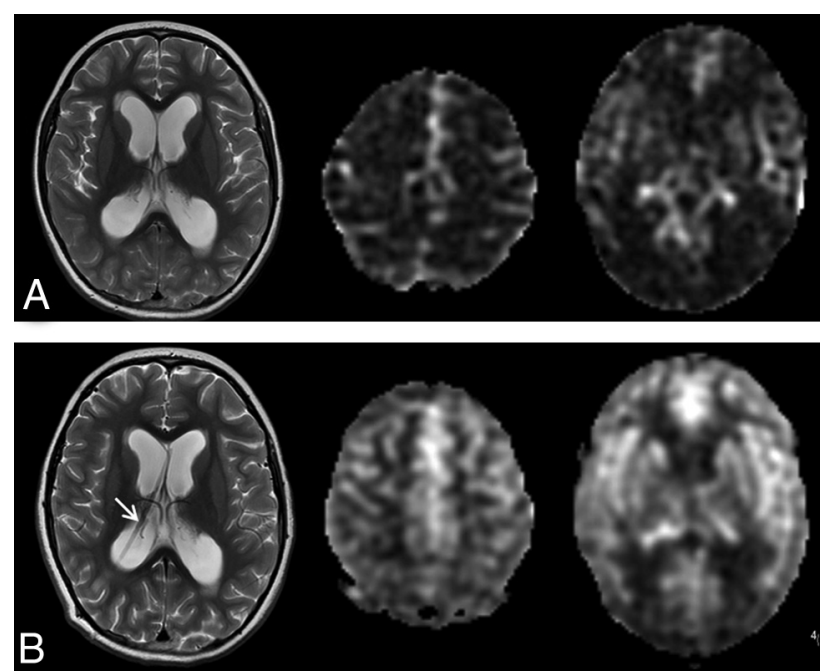

FIG 4. ASL perfusion of a 6-year-old girl presenting with hydrocephalus from a diffuse intrinsic pontine glioma. $A$, The patient presented with acute symptoms, including headache, nausea/vomiting, and somnolence. Enlarged ventricles and periventricular edema were noted and low $\mathrm{CBF}$ of $9,13,8$, and $6 \mathrm{~mL} / 100 \mathrm{~g} / \mathrm{min}$ at the cerebral vertex, putamina, thalami, and periventricular white matter, respectively. A shunt was placed a day after the MR imaging. $B$, Improved CBF $(30,40,36,27 \mathrm{~mL} / 100$ $\mathrm{g} / \mathrm{min}$ ) and resolution of acute symptoms were noted in the respective brain regions a month later. Note the shunt catheter in place (arrow) and residual ventricular enlargement and edema.

showed low hemispheric CBF (32 $\pm 6 \mathrm{~mL} / 100 \mathrm{~g} / \mathrm{min}$ ) by using ${ }^{133} \mathrm{Xe}$ CT in adults who presented with hydrocephalus after aneurysm rupture and correlative high ICP levels (36 $\pm 6 \mathrm{~mm} \mathrm{Hg})$.
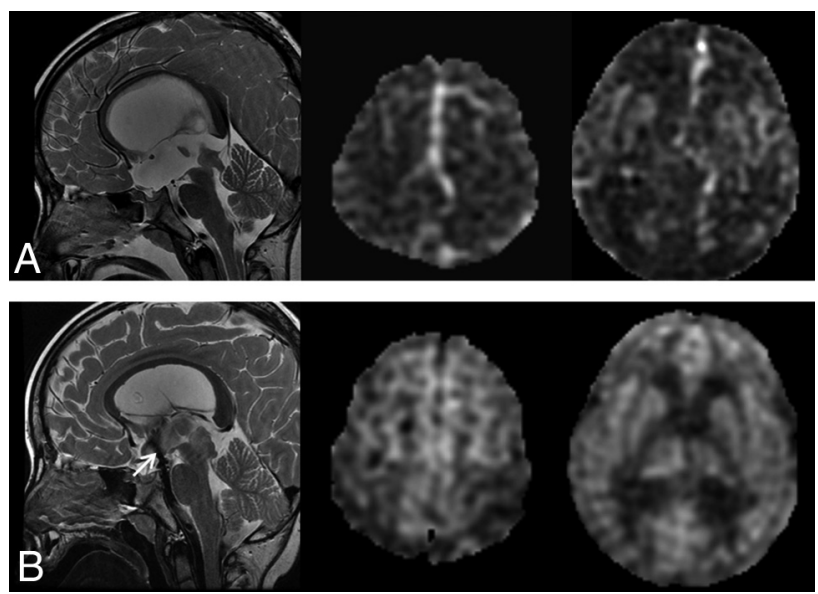

FIG 5. ASL perfusion of a 5-year-old boy presenting with acute hydrocephalus due to aqueduct obstruction from a tectal glioma. A, The patient presented with acute gait abnormality, nausea/vomiting, and somnolence. Enlarged ventricles and low global CBF of 16, 20, 17, and 8 $\mathrm{mL} / 100 \mathrm{~g} / \mathrm{mL}$ were seen at the cerebral vertex, putamina, thalami, and periventricular white matter, respectively. The patient underwent third ventriculostomy a day later and subsequently showed symptomatic relief. B, Two months later, improved CBF was seen, with CBF of $43,58,50$, and $32 \mathrm{~mL} / 100 \mathrm{~g} / \mathrm{min}$ in the respective brain regions. Note a CSF jet at the patent third ventriculostomy site (arrow).

Leliefeld et $\mathrm{al}^{23}$ also reported low CBF $(25 \pm 11 \mathrm{~mL} / 100 \mathrm{~g} / \mathrm{min})$ calculated from carotid and basilar artery flow rates by using $2 \mathrm{D}$ phase-contrast MRA in children with hydrocephalus. The lower CBF estimated in that study may be attributed to some combination of the younger age (range, 1 day to 7 months) of their cohort that might have had less robust autoregulatory mechanisms or more compressible parenchymal vessels within an immature brain, low signal due to a 2D phase-contrast MRA technique, and variable etiologies of hydrocephalus (intraventricular hemorrhage, arachnoid cyst, mucopolysaccharidosis, and various obstructive lesions). Of note, CBF of our healthy pediatric controls $(62.3 \pm 6 \mathrm{~mL} / \mathrm{min} / 100 \mathrm{~g})$ is consistent with a normal mean CBF range of $53-71 \mathrm{~mL} / \mathrm{min} / 100 \mathrm{~g}$ in children $2-19$ years of age measured by ${ }^{133} \mathrm{Xe} \mathrm{SPECT}^{34}$ but higher than ${ }^{133} \mathrm{Xe}$ CT-based mean $\mathrm{CBF}$ of $50 \mathrm{~mL} / \mathrm{min} / 100 \mathrm{~g}$ reported for healthy adults. ${ }^{33}$

$\mathrm{CBF}$ was reduced for all brain regions in patients presenting with symptomatic hydrocephalus. Studies have shown that ventriculomegaly may directly decrease CBF via mechanical distortion and reduced vessel caliber. ${ }^{35,36}$ Alternatively, reduced brain compliance and, thereby, narrow-capacitance vessels, induced by ventricular enlargement, may decrease $\mathrm{CBV}$ and $\mathrm{CBF}$, similar to a hydrodynamic mechanism proposed by Greitz. ${ }^{37,38}$ However, it is noteworthy that ventricular size inversely correlated with CBF of the cerebrum at the vertex, but not in the other brain regions. It is possible that deep gray and periventricular regions are more vulnerable to mechanical effects with pronounced CBF changes, even with mild ventriculomegaly. This finding unlikely reflects under- 

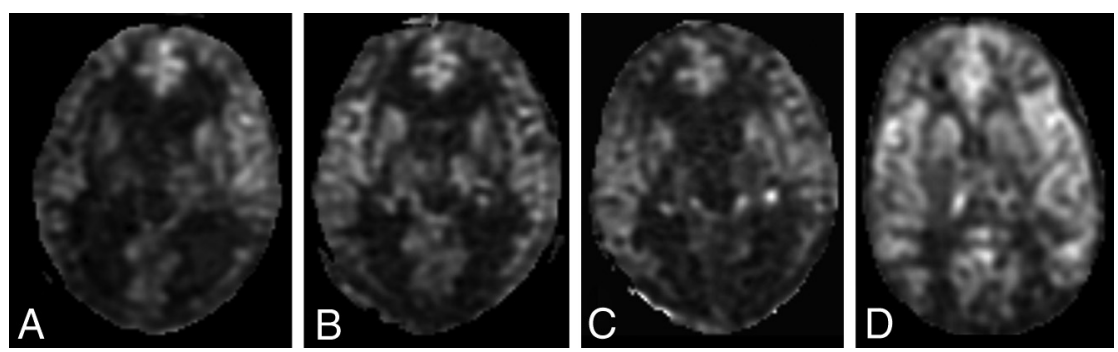

FIG 6. Serial ASL perfusion of a 10-year-old boy with hydrocephalus from medulloblastoma. $A$, The patient presented acutely with headaches and nausea/vomiting. Relatively low CBF of 29, 50, $45,20 \mathrm{~mL} / 100 \mathrm{~g} / \mathrm{min}$, compared with controls, was noted in the cerebral vertex, putamina, thalami, and periventricular white matter. The patient underwent PF tumor resection and extraventricular drain placement 2 days later. $B$, One day after the surgery, the patient continued to have high ICP despite extraventricular drain placement and showed low CBF of 22, 34, 30, and 12 $\mathrm{mL} / 100 \mathrm{~g} / \mathrm{min}$ in the respective brain regions. $C$, The symptoms continued to worsen with high ICP and further decrease in CBF of 17, 31, 18, and $10 \mathrm{~mL} / 100 \mathrm{~g} / \mathrm{min} 2$ days later. Due to extraventricular drain malfunction, a new ventriculoperitoneal shunt was subsequently placed. $D$, Several months later, the patient presented for routine surveillance of medulloblastoma with a functioning ventriculoperitoneal shunt and decreased ventricular size and was clinically asymptomatic at this time. While some perfusion alteration may be expected from interval chemotherapy and radiation, it has, nevertheless, improved, with a $\mathrm{CBF}$ of $36,44,34,21 \mathrm{~mL} / 100 \mathrm{~g} / \mathrm{min}$ in the respective brain regions.

lying regional pressure differences because no evidence for transmantle pressure gradient has been shown in hydrocephalus due to viscoelasticity of the brain substance. ${ }^{8,39}$

Concepts of hydrocephalus are complex and continue to evolve. ${ }^{8,9,37,38}$ They include the following: the conventional major CSF pathway, including the original work of Dandy and Blackfan ${ }^{40}$ on bulk flow theory and distinction of obstructive and communicating hydrocephalus; and a minor CSF pathway that describes interactions of brain compliance, CSF and arteriole pulsations, vascular capacitance/pulse pressures, and capillary absorption. ${ }^{8,9,37,40-42}$ Given the complex pathophysiology, understanding the physiology of hydrocephalus remains a challenging neurosurgical problem.

While ventricular size remains important for the work-up of hydrocephalus, prior studies have shown that it may not reliably identify abnormal ICP ${ }^{1-13,15}$ or resorptive capacity for CSF, ${ }^{11}$ particularly in cases of chronic hydrocephalus or shunt failure in which brain compliance may be altered from injury, periventricular gliosis, and other reactive changes. ${ }^{12}$ Our results suggest that ASL perfusion identifies changes in underlying tissue physiology and may, therefore, be a useful adjunct to conventional MR imaging without the need for contrast or radiation exposure.

There are a few limitations of this study. Direct CSF pressure recordings that correspond to $\mathrm{CBF}$ changes would be desirable; however, pressure measurements are not routinely obtained during resection of PF tumors or at the time of MR imaging and, therefore, were not feasible. While ASL-CBF was altered in the uncompensated state and was restored after CSF symptomatic relief in this population of pediatric patients with PF tumors, baseline perfusion metrics and deficit patterns may vary to some degree in other models of hydrocephalus, such as normal-pressure hydrocephalus of older adults; chronic, compensated, or nonprogressive hydrocephalus; and various conditions of hydrocephalus associated with prior hemorrhage/ injury, brain malformations, and metabolic disorders and congenital chromosomal disorders. However, CBF changes hydrocephalus, as a potential noninvasive method to follow changes in ICP.

\section{REFERENCES}

1. Bondurant CP, Jimenez DF. Epidemiology of cerebrospinal fluid shunting. Pediatr Neurosurg 1995;23:254-58, discussion 259

2. Simon TD, Riva-Cambrin J, Srivastava R, et al. Hospital care for children with hydrocephalus in the United States: utilization, charges, comorbidities, and deaths. J Neurosurg Pediatr 2008; $1: 131-37$

3. Eklund A, Smielewski P, Chambers I, et al. Assessment of cerebrospinal fluid outflow resistance. Med Biol Eng Comput 2007;45:719-35

4. Sundström N, Andersson K, Marmarou A, et al. Comparison between 3 infusion methods to measure cerebrospinal fluid outflow conductance. J Neurosurg 2010;113:1294-303

5. Walker CT, Stone JJ, Jacobson M, et al. Indications for pediatric external ventricular drain placement and risk factors for conversion to ventriculoperitoneal shunt. Pediatr Neurosurg 2012;48:342-47

6. Stone JJ, Walker CT, Jacobson M, et al. Revision rate of pediatric ventriculoperitoneal shunts after 15 years. J Neurosurg Pediatr 2013;11:15-19

7. Vinchon M, Rekate H, Kulkarni AV. Pediatric hydrocephalus outcomes: a review. Fluids Barriers CNS 2012;9:18

8. Rekate HL. A consensus on the classification of hydrocephalus: its utility in the assessment of abnormalities of cerebrospinal fluid dynamics. Childs Nerv Syst 2011;27:1535-41

9. Symss NP, Oi S. Theories of cerebrospinal fluid dynamics and hydrocephalus: historical trend. J Neurosurg Pediatr 2013;11:170-77

10. Preuss M, Hoffmann KT, Reiss-Zimmermann M, et al. Updated physiology and pathophysiology of CSF circulation - the pulsatile vector theory. Childs Nerv Syst 2013;29:1811-25

11. Børgesen SE, Gjerris F. Relationship between intracranial pressure, ventricular size, and resistance to CSF outflow. J Neurosurg 1987;67:535-39

12. Dahlerup B, Gierris F, Harmsen A, et al. Severe headache as the only symptom on long-standing shunt dysfunction in hydrocephalic children with normal or slit ventricles revealed by computed tomography. Childs Nerv Syst 1985;1:49-52

13. Ashley WW Jr, McKinstry RC, Leonard JR, et al. Use of rapid-se- 
quence magnetic resonance imaging for evaluation of hydrocephalus in children. J Neurosurg 2005;103(2 suppl):124-30

14. Tans JT, Poortvliet DC. Relationship between compliance and resistance to outflow CSF in adult hydrocephalus. J Neurosurg 1989;71:59-62

15. Brawanski A, Soerensen N. Increased ICP without ventriculomegaly. Diagnostic and therapeutic problems in a 1-year old boy. Childs Nerv Syst 1985;1:66-68

16. Johnston I, Paterson A. Benign intracranial hypertension. II. CSF pressure and circulation. Brain 1974;97:301-12

17. Grubb RL, Raichle ME, Phelps ME, et al. Effects of increased intracranial pressure on cerebral blood volume, blood flow, and oxygen utilization in monkeys. J Neurosurg 1975;43:385-98

18. Häggendal E, Lofgren J, Nilsson NJ, et al. Effects of varied cerebrospinal fluid pressure on cerebral blood flow in dogs. Acta Physiol Scand 1970;79:262-71

19. Jones HC, Richards HK, Bucknall RM, et al. Local cerebral blood flow in rats with congenital hydrocephalus. J Cereb Blood Flow Metab 1993;13:531-34

20. da Silva MC, Michowicz S, Drake JM, et al. Reduced local cerebral blood flow in periventricular white matter in experimental neonatal hydrocephalus-restoration with CSF shunting. J Cereb Blood Flow Metab 1995;15:1057-65

21. Shirane R, Sato S, Satao K, et al. Cerebral blood flow and oxygen metabolism in infants with hydrocephalus. Childs Nerv Syst 1992;8:118-23

22. Shinoda M, Yamaguchi T, Tanaka Y, et al. Single photon emission computerized tomography in childhood hydrocephalus. Childs Nerv Syst 1992;8:219-21

23. Leliefeld PH, Gooskens R HJM, Vincken KL, et al. Magnetic resonance imaging for quantitative flow measurements in infants with hydrocephalus: a prospective study. J Neurosurg Pediatr 2008;2:163-70

24. Wheaton AJ, Miyazaki M. Non-contrast enhanced MR angiography: physical principles. J Magn Reson Imaging 2012;36:286-304

25. Ozsarlak O, Van Goethem JW, Maes M, et al. MR angiography of the intracranial vessels: technical aspects and clinical applications. Neuroradiology 2004;46:955-72

26. Wang J, Licht DJ, Jahng GH, et al. Pediatric perfusion imaging using pulsed arterial spin labeling. J Magn Reson Imaging 2003;18:404-13

27. Wang J, Licht DJ. Pediatric perfusion MR imaging using arterial spin labeling. Neuroimaging Clin Am 2006;16:149-67, ix
28. Dai W, Garcia D, Bazelaire C, et al. Continuous flow-driven inversion for arterial spin labeling using pulsed radio frequency and gradient fields. Magn Reson Med 2008;60:1488-97

29. Buxton RB, Frank LR, Wong EC, et al. A general kinetic model for quantitative perfusion imaging with arterial spin labeling. Magn Reson Med 1998;40:383-96

30. van Gelderen P, de Awart JA, Duyn JH. Pitfalls of MRI measurements of white matter perfusion based on arterial spin labeling. Magn Reson Med 2008;59:788-95

31. Ye FQ, Berman KF, Ellmore T, et al. H2 150 PET validation of steady-state arterial spin tagging cerebral blood flow measurements in humans. Magn Reson Med 2000;44:450-56

32. Kirkpatrick M, Engleman H, Minns RA. Symptoms and signs of progressive hydrocephalus. Arch Dis Child 1989;64:124-28

33. Hayashi M, Kobayashi H, Kawano H, et al. Cerebral blood flow and ICP patterns in patients with communicating hydrocephalus after aneurysm rupture. J Neurosurg 1984;61:30-36

34. Chiron C, Raynaud C, Maziere B, et al. Changes in regional cerebral blood flow during brain maturation in children and adolescents. J Nucl Med 1992;33:696-703

35. Wozniak M, McLone DG, Raimondi AJ. Micro- and macrovascular changes as the direct cause of parenchymal destruction in congenital murine hydrocephalus. J Neurosurg 1975;43:535-45

36. Del Bigio MR. Neuropathological changes caused by hydrocephalus. Acta Neuropathol 1993;85:573-85

37. Greitz D. Radiological assessment of hydrocephalus: new theories and implications for therapy. Neurosurg Rev 2004;27:145-65, discussion 166-67

38. Greitz D. Paradigm shift in hydrocephalus research in legacy of Dandy's pioneering work: rationale for third ventriculostomy in communicating hydrocephalus. Childs Nerv Syst 2007;23:487-89

39. Stephensen H, Tisell M, Wikkelsö C. There is no transmantle pressure gradient in communicating or noncommunicating hydrocephalus. Neurosurgery 2002;50:763-71, discussion 771-73

40. Dandy WE, Blackfan KD. Internal hydrocephalus: an experimental, clinical, and pathological study. Am J Dis Child 1914;8:406-82

41. Oi S, Di Rocco C. Proposal of "evolution theory in cerebrospinal fluid dynamics" and minor pathway hydrocephalus in immature brain. Childs Nerv Syst 2006;22:662-69

42. Dandy WE. Where is cerebrospinal fluid absorbed? JAMA 1929;92: 2012-14 